\title{
Fuzzy hybrid MCDM approach for selection of wind turbine service technicians
}

\author{
Goutam Kumar Bose $^{\mathrm{a}^{*}}$ and Nikhil Chandra Chatterjee ${ }^{\mathrm{b}}$
}

${ }^{a}$ Professor, Department of Mechanical Engineering, Haldia Institute of Technology, Haldia, West Bengal University of Technology, West Bengal, India ${ }^{b}$ Assistant Professor, Department of Mechanical Engineering, Saroj Mohan Institute of Technology, Guptipara, West Bengal University of Technology, West Bengal, India

\section{H R O N I C L E}

Article history:

Received August 28, 2015

Received in revised format

November 28, 2015

Accepted December 6, 2015

Available online

December 10, 2015

Keywords:

Wind Turbine Service Technicians

(Wind techs)

MCDM

Fuzzy Set Theory

Multi Criteria Group Decision

making (MCGDM)

ARAS-F

MOORA $-F$

\begin{abstract}
A B S T R A C T
This research paper is aimed to present a fuzzy Hybrid Multi-criteria decision making (MCDM) methodology for selecting employees. The present study aspires to present the hybrid approach of Fuzzy multiple MCDM techniques with tactical viewpoint to support the recruitment process of wind turbine service technicians. The methodology is based on the application of Fuzzy ARAS (Additive Ratio Assessment) and Fuzzy MOORA (Multi-Objective Optimization on basis of Ratio Analysis) which are integrated through group decision making (GDM) method in the model for selection of wind turbine service technicians' ranking. Here a group of experts from different fields of expertise are engaged to finalize the decision. Series of tests are conducted regarding physical fitness, technical written test, practical test along with general interview and medical examination to facilitate the final selection using the above techniques. In contrast to single decision making approaches, the proposed group decision making model efficiently supports the wind turbine service technicians ranking process. The effectiveness of the proposed approach manifest from the case study of service technicians required for the maintenance department of wind power plant using Fuzzy ARAS and Fuzzy MOORA. This set of potential technicians is evaluated based on five main criteria.
\end{abstract}

\section{Introduction}

Owing to the revolution in using wind energy, maintenance of wind turbine has been a challenge to the maintenance engineering team. A wind farm generates rated power output for supplying to the public or industrial service, without any interruption. Wind turbine service technicians also known as wind techs play one of the most pivoted role for the same. Wind techs inspect, diagnose problems, repair parts or components of wind turbines. Maintenance work is carried out on the equipment for resolving electrical, mechanical, and hydraulic malfunctioning. Therefore it is imperative that the selection and recruitment of efficient wind turbine technician can increase the useful life of turbines. Personnel selection is the process of examining the applicants by evaluating their KSAPs (knowledge, skill, ability * Corresponding author.

E-mail address: gkbose@yahoo.com (G. K. Bose) 
and personalities) for a definite job(s) and identifying the suitable candidate(s) for the organization's requirements.

Multi-criteria decision making (MCDM) technique is applied where a lot of conflicting criteria are involved and complex decisions are taken. MCDM methods may be classified according to the number of decision makers involved in the decision making process, i.e., single decision maker MCDM method and group decision making MCDM method i.e., MCGDM method. In MCGDM a committee of decision makers is formed to rank all the alternatives more rationally by aggregating the judgment of each alternative by each decision maker of their respective knowledge, experience and preference for a finite set of alternatives (Liang and Pang 2012). Another way to classify the MCDM methods with respect to nature of data available is Classical MCDM and Fuzzy MCDM (FMCDM).

In case of selection of Wind Techs, not only KSAPs are examined but physical ability or strength and as well as mental fitness are important criteria for the same. The quality factors of a person which includes personality, leadership quality, teamwork ability, self confidence, physical ability, mental fitness, oral communication skills and past experiences, cannot be assessed by crisp values. Fuzzy set theory is immensely useful to transform the inadequate, vague, non-crisp information by using linguistic variables i.e., whose values are expressed not in numbers, but in words and triangular fuzzy numbers (TFNs) Zadeh (1965). A candidate undergoes through several tests during the selection process. A test is a sample of aspect of individuals' performance by which the selectors can compare amongst the candidates and assess the individuals. Depending on the organization's specific targets, strategy and nature of work the selection process becomes complex. Since many conflicting criteria persist, MCDM is the best-suited technique for handling this complexity. It is a challenging task to judge the entire criterion by a single decision maker (DM). Therefore multiple decision makers (DMs) are engaged in the process, which formulates the MCGDM problem.

Some of the past research works are presented here. Brauers and Zavadskas (2006) introduced MOORA (Multi-Objective Optimization on basis of Ratio Analysis) associated with reference point theory to choose the best alternative amongst a collection of feasible alternatives. This approach is employed by various researchers to solve the decision making problems. Brauers, et al. (2008) used MOORA method to rank the contractors. Zadeh (1965) introduced Fuzzy logic, which can consider the uncertainty and resolve the problems where there are no sharp boundaries and precise values. Afshari, et al. (2010) applied Simple Additive Weighting (SAW) method to select the best five personnel for a telecommunication company. Chen, (2000) applied Technique for Order Preference by Similarity to Ideal Solution (TOPSIS) for group decision-making under fuzzy environment for selecting a system analysis engineer of a software company. Chen et al. (2011) present a Serbian: VIseKriterijumsa Optimizacija I Kompromisno Resenje, (VIKOR) means: Multi-criteria Optimization and Compromise Solution based methodology with 2-Tuple linguistic variable to select an engineer for a semi-conduct industry using multiple decision makers. El-Santawy (2012) applied VIKOR method for solving personnel selection problem for training for a multi-national telecommunications company. Baležentis et al. (2012) used fuzzy Multiplicative form of Multi Objective Optimization on basis of Ratio Analysis (MULTIMOORA) with linguistic reasoning and group decision-making (MULTIMOORA-FG) by aggregating the individual assessments of the decision-makers to perform a more robust personnel selection. Ding (2012) developed a fuzzy MCDM model to effectively select middle managers for the global shipping carrier-based logistics service providers.

Blanco and Rodrigues (2009) estimated the direct wind energy employment in all EU countries on their research work. They analyzed various aspects, mainly gender distribution, company profiles and also the shortage of skilled workers by surveying wind energy companies. There is a scarcity of specialists in wind energy sector i.e., project managers, engineers and O\&M technicians etc. Bobrow (2003) developed a general framework, using of psychological tests for employee based on sound practices and legal precedents selection to avoid any unfair means in personnel selection. He framed suitable 
selection methods that are most likely to be correlated with performance for the given job, based on the results of job analysis. Robertson and Smith (2001) presented good reviews on personnel selection studies. They investigated the responsibility of job analysis, contemporary models of work performance, and set of criteria used in personnel selection process. Liang and Wang (1994) formed a fuzzy multi-criteria decision making algorithm for personnel selection by employing fuzzy ranking methods to establish the most appropriate candidate. Karsak (2001) offered a fuzzy MCDM methodology based on ideal and anti-ideal solutions for the finding of the best candidate. Chen et al. (2005) applied FAHP to determine the weighting of subjective judgments for expatriate candidates to decide the relative importance for criteria of expatriate assignments that offers guidelines for managers concerned with a successful emigrant assignment program. Gibney and Shang (2007) presented the use of the analytical hierarchy process (AHP) in the personnel selection process. Zavadskas et al. (2008) applied grey relations methodology for defining the utility of alternatives and a multiple criteria method of COmplex PRoportional ASsessment of alternatives with Grey relations (COPRAS-G) for the selection of project managers.

Güngör et al. (2009) planned a personnel selection system based on Fuzzy Analytic Hierarchy Process (FAHP) to evaluate the finest and satisfactory personnel dealing with the rating of both qualitative and quantitative criteria. Kelemenis and Askounis (2009) put forward a fuzzy TOPSIS based MCDM approach to deal with group decision making under fuzzy environment for selection of a middle level consulting manager. Datta et al. (2009) used COPRAS-G method for quality evaluation and selection of a suitable research guide or supervisor analyzing the criteria that influence the efficacy of a quality researcher while directing his/her scholar towards a positive and successful research outcome. Dursun and Karsak (2010) presented a fuzzy multi-criteria decision making (FMCDM) algorithm using the principles of fusion of fuzzy information, 2-tuple linguistic representation model. TOPSIS method is applied to select suitable personnel for a manufacturing company with an illustrative example based on an earlier work by Liang and Wang (1994).

Pramanik and Mukhopadhyaya (2011) developed a fuzzy multi criteria group making method with Grey Relational Analysis (GRA) for teacher selection in higher education. They used Intuitionistic fuzzy weighted averaging operator to aggregate individual opinions of decision makers into a group opinion. Kelemenis et al (2011) applied fuzzy TOPSIS for group decision making to select middle level manager in a large IT Greek firm. They introduced three new concepts, i,e., the relative importance of the decision makers per criterion, secondly, the similarity-proximity degree among the decision makers and the veto thresholds, for the better description of the problem and to support the process. Keršuliene and Turskis (2011) proposed a fuzzy MCDM model using ARAS-F and step-wise weight assessment ratio analysis (SWARA) technique for architect selection problem.

Haghighi et al. (2012) proposed an employee evaluation and selection approach based on fuzzy multiple attribute decision making (MADM) through triangular fuzzy numbers to evaluate the best adequate employee dealing with the rating of both qualitative and quantitative criteria. Dadelo et al. (2012) offers a model for selection of elite security personnel based on expert evaluation method to determine criteria weights known as Dadelo's methodology and on ARAS (Additive Ratio Assessment) method to aggregate criteria values. Rouyendegh and Erkan (2013), applied fuzzy ELECTRE method with group decision making to select academic staff. Aggarwal (2013) presents a new AHP weighted fuzzy linear programming model (AHP-FLP) for personnel selection for an Indian IT industry based on multiple criteria then compared with the classical AHP method.

The objective of the present paper is to select the suitable Wind Turbine Technicians for a wind farm taking into account the considerable criteria initially by using ARAS-F (Fuzzy - $\underline{\text { Additive }}$ Ratio

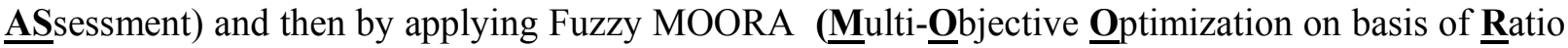

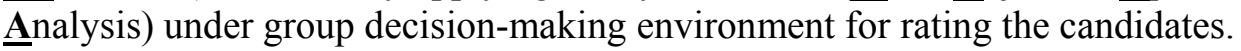




\subsection{Fuzzy Set Theory}

Fuzzy set theory is used to handle immeasurable or numerically inexpressible information and to make all the information uniform. Fuzzy set theory is initially introduced by Zadeh (1965) to resolve the uncertainty arises in decision making problem by applying it. Sometimes ambiguity, vagueness and ambivalence in judgments of decision maker(s) makes the problem difficult to measure exact numerical value, so the crisp data are inadequate to model an uncertain MCDM problem. Utilization of linguistic assessments of weights of the criteria in the problem are more realistic approach instead of numerical values. The decision matrix can be converted into a fuzzy decision matrix and a weighted normalized fuzzy decision matrix. The methodology is simple and easy to compute in any spreadsheet software.

In a cosmos of conversation $\mathrm{X}$, a fuzzy set $\hat{d}$ is characterized by a membership function $\mu_{\dot{\hat{d}}}(x)$, i.e., degree of membership of $\mathrm{x}$ in $\hat{d}$ which maps each element $\mathrm{x}$ in $\mathrm{X}$ to a real number in the interval $[0,1]$. A triangular fuzzy number (TFN), $\widehat{d}$ can be defined as a triplet $\left(d_{1}, d_{2}, d_{3}\right)$ and the membership function is defined (Dubois and Prade 1978, Keufmann and Gupta 1991) as shown by Eq. (1).

$$
\mu_{\tilde{d}}(x)= \begin{cases}0, & x \leq d_{1} \\ \frac{x-d_{1}}{d_{2}-d_{1}}, & \mathrm{~d}_{1} \leq x \leq d_{2} \\ \frac{d_{3}-x}{d_{3}-d_{2}}, & \mathrm{~d}_{2} \leq x \leq d_{3} \\ 0, & x>\mathrm{d}_{3}\end{cases}
$$

The fuzzy membership functions have been defined in figure 1 . The first point $\left(d_{1}\right)$ marks the location where the membership function begins to rise above 0 . The second point $\left(d_{2}\right)$ indicates where it reaches 1 and the third point $\left(d_{3}\right)$ marks where it returns to 0.

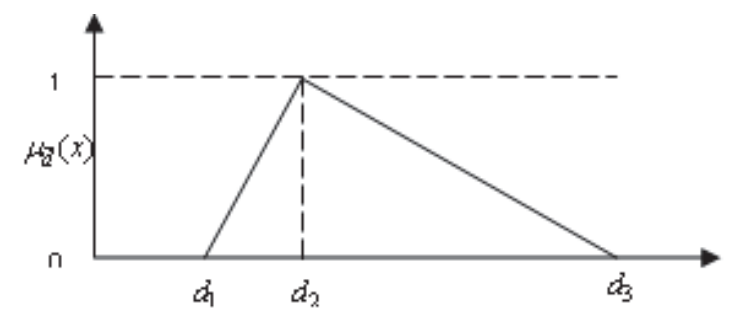

Fig. 1. Membership function of a triangular fuzzy number

The conversion technique of fuzzy number into non-fuzzy number, i.e., crisp value is known as defuzzyfication. In this paper 'centroid of area' technique for determining Best Non-fuzzy Performance (BNP) value is applied.

$$
B N P=\frac{\left[\left(d_{3}-d_{1}\right)-\left(d_{2}-d_{1}\right)\right]}{3}+d_{1}
$$

\subsection{ARAS-F}

Additive Ratio ASsessment (ARAS) (Zavadskas and Turskis, 2010 \& Turskis and Zavadskas, 2010) method logically compares the ratio of the sum of normalized and weighted values of criteria, which describe available alternative, to the sum of the values of normalized. The weighted criterion, that describes the optimal alternative, is degree of optimality, which is attained by the alternative under comparison. In ARAS method, the utility function value of a feasible alternative is directly proportional to the relative effect of values and weights of the main criteria. The steps for solving the problems are followed using algorithm by Chatterjee and Bose, 2013: 
Step1: Initialization of decision-making, i.e., the number of feasible alternatives (m), determination of the evaluation criteria $(n)$, numbers of decision makers involved.

Step 2: Choosing the linguistic ratings for criteria and alternatives from Table 1.

Table 1

Linguistic terms for criteria \& alternatives

\begin{tabular}{cccc}
\hline Criteria & \multicolumn{2}{c}{ Alternatives } \\
\hline Linguistic terms & Fuzzy number & Linguistic terms & Fuzzy number \\
\hline Very High (VH) & $(0.9,1.0,1.0)$ & Very Good (VG) & $(0.9,1.0,1.0)$ \\
High (H) & $(0.7,0.9,1.0)$ & Good (G) & $(0.7,0.9,1.0)$ \\
Moderate High (MH) & $(0.5,0.7,0.9)$ & Medium Good (MG) & $(0.5,0.7,0.9)$ \\
Moderate (M) & $(0.3,0.5,0.7)$ & Medium (M) & $(0.3,0.5,0.7)$ \\
Moderate Low (ML) & $(0.1,0.3,0.5)$ & Medium Poor (MP) & $(0.1,0.3,0.5)$ \\
Low (L) & $(0.0,0.1,0.3)$ & Poor (P) & $(0.0,0.1,0.3)$ \\
Very Low (VL) & $(0.0,0.0,0.1)$ & Very Poor (VP) & $(0.0,0.0,0.1)$ \\
\hline
\end{tabular}

Step 3: Formation of the decision matrix: The fuzzy decision-making matrix is formed as shown in Eq. (3)

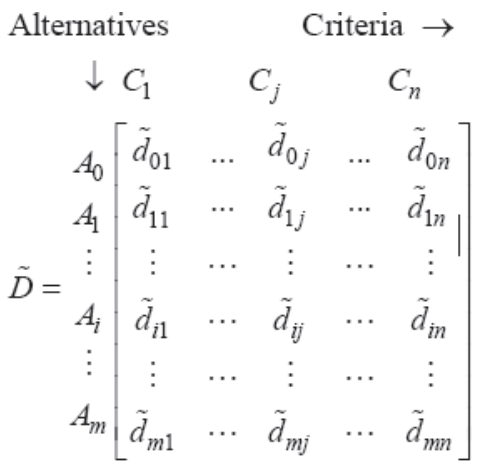

Step 4: Defuzzifying the fuzzy decision matrix using the 'centroid of the area method'. Defuzzification of the fuzzy decision matrix into crisp values is prepared by using the Eq. (2).

Step 5: Normalization of defuzzified decision matrix.

Step 6: Formation of the Weighted Normalized Decision Matrix

Step 7: Determining values of optimality function of $i^{\text {th }}$ alternative $\left(S_{i}\right)$, i.e., the sum of criteria measures. The biggest value is the best and the lowest one is the worst.

Step 8: Ranking: The ranking of the alternatives can be determined according to the value $S_{i}$. Then, the alternative with the highest score is selected as the preferred (best) one.

Step 9: Computation of degree of the alternative utility $\left(K_{i}\right)$

\subsection{MOORA-F}

MOORA (Multi-Objective Optimization on basis of Ratio Analysis) was introduced by Brauers and Zavadskas in 2006. It is a multi-criteria or multi attribute optimization process of simultaneously optimizing two or more conflicting attributes (objectives) subject to certain constraints. MOORA is related with reference point theory which assists to decide the best substitute amid a pool of viable 
choices. Here a comparison of the variant can be analyzed, with the ideal alternative i.e., the demand of the organization.

The steps of the method are as follows:

Step 1: Initialization of decision-making: Generation of feasible alternatives (m), Determination of the evaluation criteria (n), selecting a group of decision makers $(\mathrm{k})$.

Step 2: Choosing the linguistic ratings for criteria and alternatives: The importance weights of various criteria and alternatives are considered as linguistic variables. These linguistic variables can be expressed in positive triangular fuzzy numbers as Tables 1 and 2.

Table 2

Linguistic terms for alternative

\begin{tabular}{ll}
\hline Linguistic terms & Fuzzy number \\
\hline Very Good (VG) & $(0.9,1.0,1.0)$ \\
Good (G) & $(0.7,0.9,1.0)$ \\
Medium Good (MG) & $(0.5,0.7,0.9)$ \\
Medium (M) & $(0.3,0.5,0.7)$ \\
Medium Poor (MP) & $(0.1,0.3,0.5)$ \\
Poor (P) & $(0.0,0.1,0.3)$ \\
Very Poor (VP) & $(0.0,0.0,0.1)$ \\
\hline
\end{tabular}

Step 3: Formation of the decision matrix: The fuzzy group decision matrix is formed as in Eq. (4)

$$
\begin{aligned}
& \text { A lternatives } \quad \text { Criteria } \rightarrow \\
& \downarrow \begin{array}{cccc}
C_{1} & C_{j} & C_{n}
\end{array}
\end{aligned}
$$

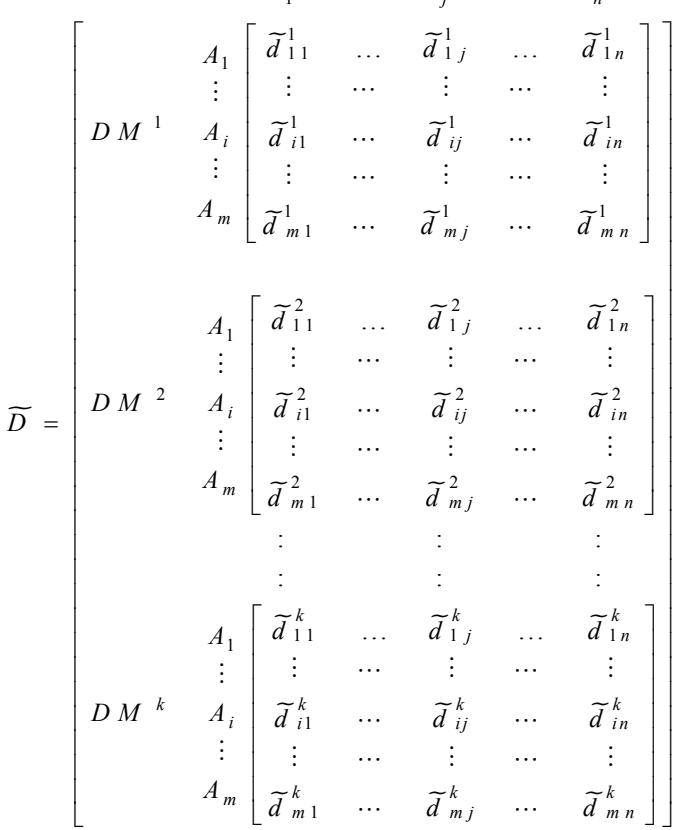

where, $\tilde{d}_{i j}^{k}=d_{i j_{1}}^{k}, d_{i j_{2}}^{k}, d_{i j_{3}}^{k}$

The weight of each criterion is calculated by summing the assigned weights by experts and then dividing the sum by the number of experts as in Eq. (5), as follows:

$$
\widetilde{D}=\left[\tilde{d}_{i j 1}, \tilde{d}_{i j 2}, \tilde{d}_{i j 3}\right] \text {, where } \tilde{d}_{i j}=\frac{1}{k}\left[\tilde{d}_{i j}{ }^{1}+\tilde{d}_{i j}{ }^{2}+\tilde{d}_{i j}{ }^{3}+\ldots \ldots . .+\tilde{d}_{i j}{ }^{k}\right]
$$

Step 3: Defuzzifying the fuzzy decision matrix: 
Defuzzification of the fuzzy decision matrix into crisp values is done by using the Eq. (2).

Step 4: Normalization of defuzzified decision matrix $(\bar{D})$ :

The objective of normalization is to obtain comparable scales of criteria values. The normalization of criteria values is not compulsory, but it may be required sometimes. In order to avoid the difficulties caused by different dimensions of the criteria values, the ratio to the optimal value is used. (Zavadskas and Turskis, 2008). The normalized value $\bar{d}_{i j}$ is calculated using the Eq. (6);

$$
\bar{d}_{i j}=\frac{d_{i j}}{\sqrt{\sum_{i=1}^{m}\left(d_{i j}\right)^{2}}} \quad(1 \leq i \leq m, \quad 1 \leq j \leq n)
$$

Step 5: Computation of the criteria weight:

Defuzzify the fuzzy weight of each criterion $\left(w_{1}, w_{2}, w_{3}\right)$ into crisp values $\left(w_{j}\right)$ by using the Eq. (7).

$$
w_{j}=\frac{\left[\left(w_{3}-w_{1}\right)-\left(w_{2}-w_{1}\right)\right]}{3}+w_{1}
$$

Compute of the Weight $q_{j}$ of $j^{\text {th }}$ criterion is as follow.

$$
q_{j}=\frac{w_{j}}{\sum_{j=i}^{n} w_{j}}
$$

Step 6: Formation of the Weighted Normalized Decision Matrix $(\widehat{D})$ : The weighted normalized decision value $(\hat{d})$ is calculated using Eq. (9).

$$
\hat{d}_{i j}=\bar{d}_{i j} \times q_{j} \quad(1 \leq i \leq m, \quad 1 \leq j \leq n)
$$

Step 7: Computations of sum of benefit criteria measures $\left(S_{B}\right)$ and sum of non-benefit criteria measures $\left(S_{N B}\right)$ : The sum of benefit criteria measures $\left(S_{B}\right)$ can be calculated by following equation.

$$
S_{B_{i}}=\sum_{j \in B} \hat{d}_{i j}
$$

The sum of non-benefit criteria measures $\left(S_{N B}\right)$ can be calculated by the equation,

$$
S_{N B_{i}}=\sum_{j \in N B} \hat{d}_{i j}
$$

Step 8: Computation of composite score $\left(C S_{i}\right)$ : The composite score can be calculated from following equation,

$$
C S_{i}=S_{B}-S_{N B}
$$

where $C S_{i}$ is composite score of alternative $i$. The value of $C S_{i}$ may be positive, negative or zero. The best alternative is one which is associated with highest $C S_{i}$ value and the worst alternative is one which is associated with the lowest $C S_{i}$ value. 
Step 9: Ranking: Ranking of products in descending order of composite scores $\left(C S_{i}\right)$. Then, the alternative with the highest score is selected as the preferred (best) one.

\section{An elaborated example: Selection of Wind Turbine Service Technicians}

The work responsibilities of wind turbine service technicians as per Hamilton and Liming (2010) are as follows:

a) Repairing and preventive maintenance of wind turbines, underground transmission systems, wind fields substations.

b) Climbing up the wind turbine towers for inspection, maintenance or repair equipment, especially for nacelles.

c) Cleaning and lubricating the shafts, bearings, and gears in nacelle regularly.

d) Reporting about the notified problems during the inspection.

e) Preparing the schedule for repair.

f) At the time of repairing they might need a new component to replace, if so, they must drive to the store.

g) They have to carry the new parts while climbing up to where it is installed.

h) Ordering spare parts.

i) Maintaining a proper inventory of parts needed for repairs

j) Responsible for administration of the site.

In order to recruit the wind turbine technicians the organization formulates a committee of selectors. The selectors take care that the post needs a highly professional approach for providing quality services. So, five numbers of criteria and each criterion have some sub-criteria are developed. For proper selection they also prepare a guideline for each sub-criterion, which are tabulated in Table 3 below.

Table 3

Criteria and sub - criteria with respective weights

\begin{tabular}{|c|c|c|c|}
\hline Criteria & Sub-criteria & Weight $\left(q_{j}\right)$ & Test No. \\
\hline \multirow{4}{*}{ C1 Physical Ability } & C11 Traveling ability & 0.248 & \multirow{4}{*}{ Test 1} \\
\hline & C12 Ability to climb up \& down from ladders, scaffolds & 0.276 & \\
\hline & C13 Ability to perform strenuous job & 0.276 & \\
\hline & Weight lifting \& moving ability & 0.200 & \\
\hline & & $\Sigma q_{j}=1.000$ & \\
\hline \multirow{4}{*}{ C2 Basic Criteria } & C21 Language Skill i.e., report writing, manual reading and analyzing, & 0.121 & \multirow{4}{*}{ Test 2} \\
\hline & C22 Mathematical Skill & 0.150 & \\
\hline & Reasoning Ability & 0.121 & \\
\hline & Computer Knowledge & 0.168 & \\
\hline & & $\Sigma q_{j}=1.000$ & \\
\hline \multirow{3}{*}{ C3 Technical Criteria } & C31 Technical Knowledge \& Problem Sensitivity & 0.168 & \multirow{3}{*}{ Test 3} \\
\hline & Material management & 0.121 & \\
\hline & Safety conciseness & 0.150 & \\
\hline & & $\Sigma q_{j}=1.000$ & \\
\hline \multirow{6}{*}{ C4 Personal Quality } & C41 Educational Qualification & 0.178 & \multirow{6}{*}{ Test 4} \\
\hline & C42 Past Experience & 0.199 & \\
\hline & C43 Leadership Quality & 0.144 & \\
\hline & C44 Ability to work in a team & 0.178 & \\
\hline & C45 Self confidence & 0.199 & \\
\hline & Integrity and Honesty & 0.103 & \\
\hline & & $\Sigma \mathrm{q}_{j}=1.000$ & \\
\hline \multirow{8}{*}{ C5 Medical Criteria } & Free from obesity and major operations within last six months & 0.160 & \multirow{7}{*}{ Test 5} \\
\hline & Diabetic fitness & 0.178 & \\
\hline & Neuro-fitness i.e., free from Acrophobia and vertigo & 0.160 & \\
\hline & C54 Fitness of cardiovascular system & 0.160 & \\
\hline & C55 Respiratory fitness & 0.160 & \\
\hline & Visual Acuity & 0.092 & \\
\hline & Hearing & 0.092 & \\
\hline & & $\Sigma \mathrm{q}_{j}=1.000$ & \\
\hline
\end{tabular}


The selection procedure is conducted by the following steps:

(i) The candidates submitting valid application will be called for Physical Fitness Test first. (Test 1)

(ii) The candidates, who will qualify in the physical endurance test, will be considered for 'Written Test'. The Written Test will have objective type questions on Current Affairs, General knowledge; Basic English, Elementary Mathematics and Analytical Aptitude in multiple choices answer system. In this step there will be no elimination. (Test 2)

(iii)Then the candidates will appear for practical test. The test will be consisting of technical knowledge i.e. about part nomenclature, repair test, problem diagnosis, inventory control, safety etc. (Test 3)

(iv) Then the qualified candidates on the basis of marks obtained in 'Written Test' and 'Practical Test' will appear for a general interview. (Test 4)

(v) After interview the provisionally selected candidates will have to undergo a medical examination. (Test 5)

(vi)After the medical test a merit list of qualified candidates will be prepared on the basis of marks obtained in the entire test.

Primarily ARAS is applied for the method of selection.

\subsection{Test 1}

In this case study for an esteemed wind farm 15 number of candidates applied for the post of Wind Tech. Subsequently they appear for Test 1 . The decision weights of the criteria for selection of the best candidate are calculated using fuzzy set theory and the result is tabulated in Table 2. The decision matrix for the Test -1, in Linguistic term is tabulated in Table 3. Next the values of optimality function $\left(S_{i}\right)$ of each candidate are determined. The ranking of the candidates are to be done according to the value of ascending order of $S_{i}$. Degree of the alternative utility for every candidate $\left(K_{i}\right)$ is being computed and tabulated in Table 4 . Now the preliminary selection/elimination is performed on the basis of the composite score. In this research study the candidates who's $S_{i}$ score is below 0.05 gets eliminated.

Table 4

Decision Matrix in Linguistic Term and Result for Test 1

\begin{tabular}{lllllllll}
\hline Criteria & \multicolumn{3}{c}{ Linguistic Terms } & \multicolumn{5}{c}{ Results } \\
\hline Weights & 0.248 & 0.276 & 0.276 & 0.200 & $S_{i}$ & $K_{i}$ & Rank & Qualified \\
\hline Candidates & C11 & C12 & C13 & C14 & & & & \\
Candidate- I & VG & VG & VG & VG & 0.090 & 1.000 & & \\
Candidate-1 & G & MG & G & VG & 0.078 & 0.867 & 4 & $\sqrt{ }$ \\
Candidate-2 & M & MP & M & VP & 0.033 & 0.364 & 14 & \\
Candidate-3 & G & MP & G & G & 0.066 & 0.728 & 7 & $\sqrt{ }$ \\
Candidate-4 & M & G & MP & M & 0.051 & 0.570 & 11 & $\sqrt{ }$ \\
Candidate-5 & MP & M & G & G & 0.058 & 0.643 & 9 & $\sqrt{ }$ \\
Candidate-6 & VG & M & G & VG & 0.075 & 0.833 & 5 & $\sqrt{ }$ \\
Candidate-7 & P & MG & M & M & 0.043 & 0.483 & 12 & \\
Candidate-8 & G & VG & G & VG & 0.085 & 0.946 & 3 & $\sqrt{ }$ \\
Candidate-9 & MG & G & MG & M & 0.066 & 0.733 & 6 & $\sqrt{ }$ \\
Candidate-10 & VG & MG & M & VP & 0.054 & 0.602 & 10 & $\sqrt{ }$ \\
Candidate-11 & G & MG & M & MG & 0.064 & 0.711 & 8 & $\sqrt{ }$ \\
Candidate-12 & P & VP & MP & G & 0.027 & 0.302 & 15 & \\
Candidate-13 & VG & VG & G & VG & 0.087 & 0.972 & 1 & $\sqrt{ }$ \\
Candidate-14 & G & VG & VG & G & 0.086 & 0.954 & 2 & $\sqrt{ }$ \\
Candidate-15 & MP & MP & M & M & 0.037 & 0.407 & 13 & \\
\hline
\end{tabular}




\subsection{Test 2 and Test 3}

The qualified candidates of Test 1 turn up for Test 2 and then Test 3 . The decision weight of the criteria for Test- $2 \& 3$ are tabulated in Table 2 . The results of these tests are tabulated in Table 5. Here the candidate whose composite score is below 0.065 is eliminated.

Table 5

Decision Matrix in Linguistic Term and Result for Tests 2 and 3

\begin{tabular}{|c|c|c|c|c|c|c|c|c|c|c|c|}
\hline \multirow[b]{2}{*}{ Weight } & \multicolumn{7}{|c|}{ Linguistic Terms } & \multirow[t]{2}{*}{$S_{i}$} & \multirow[t]{2}{*}{$K_{i}$} & \multirow[t]{2}{*}{ Rank } & \multirow[t]{2}{*}{ Qualified } \\
\hline & 0.121 & 0.150 & 0.121 & 0.168 & 0.168 & 0.121 & 0.150 & & & & \\
\hline & $\mathrm{C} 23$ & $\mathrm{C} 24$ & $\mathrm{C} 25$ & $\mathrm{C} 26$ & $\mathrm{C} 31$ & $\mathrm{C} 32$ & $\mathrm{C} 33$ & & & & \\
\hline Candidate-I & VG & VG & VG & VG & VG & VG & VG & 0.114 & 1.000 & & \\
\hline Candidate-1 & G & VG & G & VG & VG & VG & G & 0.109 & 0.958 & 1 & $\sqrt{ }$ \\
\hline Candidate-3 & VG & G & VG & VG & VG & MG & VG & 0.109 & 0.953 & 2 & $\sqrt{ }$ \\
\hline Candidate-4 & M & VG & G & G & G & MG & MG & 0.093 & 0.815 & 4 & $\sqrt{ }$ \\
\hline Candidate-5 & M & $\mathrm{P}$ & M & MG & G & M & M & 0.063 & 0.557 & 9 & \\
\hline Candidate- 6 & MP & M & $\mathrm{P}$ & M & MG & G & $\mathrm{G}$ & 0.065 & 0.574 & 8 & $\sqrt{ }$ \\
\hline Candidate-8 & VP & M & M & M & MG & VG & G & 0.068 & 0.595 & 7 & $\sqrt{ }$ \\
\hline Candidate-9 & $\mathrm{G}$ & VG & G & G & M & MG & $\mathrm{M}$ & 0.088 & 0.775 & 5 & $\sqrt{ }$ \\
\hline Candidate-10 & VG & G & VG & G & $\mathrm{P}$ & MP & MP & 0.074 & 0.646 & 6 & $\sqrt{ }$ \\
\hline Candidate-11 & $\mathrm{G}$ & VG & G & VG & VG & $\mathrm{G}$ & MG & 0.105 & 0.921 & 3 & $\sqrt{ }$ \\
\hline Candidate-13 & M & MP & $P$ & M & $\mathrm{P}$ & M & G & 0.049 & 0.431 & 11 & \\
\hline Candidate-14 & $\mathrm{P}$ & M & M & $\mathrm{P}$ & $\mathrm{G}$ & $\mathrm{G}$ & MG & 0.062 & 0.540 & 10 & \\
\hline
\end{tabular}

\subsection{Test 4}

Next the qualified candidates arrive for a general interview (Test 4). Three selectors are appointed to conduct the selection process. The decision weight of the criteria for selection for Test 4 is tabulated in Table 2. The group decision matrix for the Test 4, in Linguistic term is tabulated in Table 6. The candidate whose $S_{i}$ value is below 0.095 gets eliminated.

Table 6

Decision Matrix for Test 4 in Linguistic Term

\begin{tabular}{|c|c|c|c|c|c|c|c|c|c|c|c|}
\hline \multirow[b]{2}{*}{ Candidates } & \multicolumn{7}{|c|}{ Linguistic Terms } & \multirow[t]{2}{*}{$S_{i} \quad I$} & $i \quad$ Rank & \multicolumn{2}{|c|}{ Qualified } \\
\hline & Decision Makers & C 41 & $\mathrm{C} 42$ & C 43 & C 44 & C 45 & $\mathrm{C} 46$ & & & & \\
\hline Weight & & 0.178 & 0.199 & 0.144 & 0.178 & 0.199 & 0.103 & \multirow{4}{*}{0.150} & \multirow{4}{*}{1.000} & & \\
\hline \multirow{3}{*}{ Candidate-U } & DM 1 & VG & VG & VG & VG & VG & VG & & & & \\
\hline & DM 2 & VG & VG & VG & VG & VG & VG & & & & \\
\hline & DM 3 & VG & VG & VG & VG & VG & VG & & & & \\
\hline \multirow{3}{*}{ Candidate-1 } & DM 1 & $\mathrm{G}$ & $\mathrm{M}$ & $\mathrm{MG}$ & $\mathrm{G}$ & $\mathrm{MG}$ & $\mathrm{M}$ & \multirow{3}{*}{0.105} & \multirow{3}{*}{0.701} & & \\
\hline & DM 2 & $\mathrm{G}$ & $\mathrm{G}$ & $\mathrm{MG}$ & MG & $\mathrm{M}$ & $\mathrm{M}$ & & & 4 & $\sqrt{ }$ \\
\hline & DM 3 & $\mathrm{G}$ & $\mathrm{G}$ & M & M & M & MG & & & & \\
\hline \multirow{3}{*}{ Candidate-3 } & DM 1 & VG & $\mathrm{M}$ & G & $\mathrm{G}$ & MG & $\mathrm{G}$ & \multirow{3}{*}{0.125} & \multirow{3}{*}{0.836} & \multirow{3}{*}{2} & \multirow{3}{*}{$\sqrt{ }$} \\
\hline & DM 2 & VG & MG & VG & G & G & G & & & & \\
\hline & DM 3 & VG & $\mathrm{MG}$ & $\mathrm{G}$ & MG & MG & $\mathrm{G}$ & & & & \\
\hline \multirow{3}{*}{ Candidate-4 } & DM 1 & $\mathrm{G}$ & $\mathrm{G}$ & $\mathrm{G}$ & $\mathrm{G}$ & $\mathrm{G}$ & $\mathrm{G}$ & \multirow{3}{*}{0.127} & \multirow{3}{*}{0.850} & \multirow{3}{*}{1} & \multirow{3}{*}{$\sqrt{ }$} \\
\hline & DM 2 & VG & MG & MG & $\mathrm{G}$ & VG & MG & & & & \\
\hline & DM 3 & $\mathrm{G}$ & $\mathrm{G}$ & $\mathrm{M}$ & $\mathrm{MG}$ & $\mathrm{G}$ & MG & & & & \\
\hline \multirow{3}{*}{ Candidate-6 } & DM 1 & $\mathrm{G}$ & $\mathrm{M}$ & $\mathrm{G}$ & $\mathrm{MG}$ & $\mathrm{M}$ & $\mathrm{M}$ & \multirow{3}{*}{0.091} & \multirow{3}{*}{0.607} & \multirow{3}{*}{7} & \\
\hline & DM 2 & M & M & MG & M & MG & M & & & & \\
\hline & DM 3 & $\mathrm{M}$ & MG & MG & $\mathrm{M}$ & MP & MG & & & & \\
\hline \multirow{3}{*}{ Candidate- 8} & DM 1 & VG & $\mathrm{G}$ & $\mathrm{VG}$ & $\mathrm{G}$ & $\mathrm{M}$ & $\mathrm{MG}$ & \multirow{3}{*}{0.124} & \multirow{3}{*}{0.825} & & \\
\hline & DM 2 & $\mathrm{G}$ & $\mathrm{G}$ & $\mathrm{G}$ & $\mathrm{G}$ & MG & $\mathrm{M}$ & & & 3 & $\sqrt{ }$ \\
\hline & DM 3 & $\mathrm{G}$ & $\mathrm{G}$ & $\mathrm{G}$ & VG & MG & $\mathrm{M}$ & & & & \\
\hline & DM 1 & $M$ & MG & MG & $\mathrm{M}$ & M & MG & & & & \\
\hline Candidate-9 & DM 2 & $\mathrm{G}$ & G & $\mathrm{M}$ & M & $\mathrm{M}$ & MG & 0.085 & 0.565 & 8 & \\
\hline & DM 3 & $\mathrm{M}$ & MG & $\mathrm{M}$ & $\mathrm{P}$ & MP & MP & & & & \\
\hline & DM 1 & $\mathrm{G}$ & $\mathrm{G}$ & $\mathrm{MG}$ & $\mathrm{M}$ & $\mathrm{MG}$ & $M$ & 0098 & 0655 & & \\
\hline Candidate-10 & DM 2 & VG & $\mathrm{G}$ & MG & MG & M & M & 0.098 & 0.655 & 5 & $\sqrt{ }$ \\
\hline & DM 3 & G & MG & M & MP & $\mathrm{P}$ & MG & & & & \\
\hline & DM 1 & M & M & $\mathrm{M}$ & MG & $\mathrm{M}$ & MG & & & & \\
\hline Candidate-11 & DM 2 & G & MG & M & M & MG & MG & 0.096 & 0.639 & 6 & $\sqrt{ }$ \\
\hline & DM 3 & $\mathrm{M}$ & $\mathrm{M}$ & $\mathrm{MG}$ & $\mathrm{M}$ & MG & M & & & & \\
\hline
\end{tabular}




\subsection{Test 5}

Lastly the qualified candidates appear for medical fitness test (Test 5). The decision weights of the criteria for selection of the best candidate are tabulated in Table 2. The decision matrix for the Test 5, in Linguistic terms and result of the test are tabulated in Table 7. Here the candidates whose $S_{i}$ value is below 0.1 are eliminated.

Table 7

Decision Matrix for Test 5 in Linguistic Terms

\begin{tabular}{llllllllllll}
\hline Candidates & C51 & C52 & C53 & C54 & C55 & C56 & C57 & $S_{i}$ & $K_{i}$ & Rank & Qualified \\
\hline Candidate-U & VG & VG & VG & VG & VG & VG & VG & 0.177 & 1.000 & & \\
Candidate-1 & M & G & VG & G & G & M & G & 0.143 & 0.806 & 3 & $\sqrt{ }$ \\
Candidate-3 & M & P & MP & M & M & G & G & 0.082 & 0.459 & 6 & \\
Candidate-4 & G & G & M & G & G & M & MG & 0.133 & 0.752 & 4 & $\sqrt{ }$ \\
Candidate-8 & VG & VG & G & VG & G & G & G & 0.167 & 0.940 & 1 & $\sqrt{ }$ \\
Candidate-10 & G & G & G & G & G & G & G & 0.159 & 0.897 & 2 & $\sqrt{ }$ \\
Candidate-11 & G & G & MG & M & MG & M & M & 0.134 & 0.757 & 5 & $\sqrt{ }$ \\
\hline
\end{tabular}

\subsection{Final Result}

Then the final merit list of five suitable candidates is prepared on the basis of $S_{i}$ value in all the tests. The candidate, who will make all-round performance stood first in merit list, is tabulated in Table 8 below.

Table 8

Final Result

\begin{tabular}{lcccccc}
\hline Candidates & $\mathrm{S}_{i 1}$ & $\mathrm{~S}_{i(2+3)}$ & $\mathrm{S}_{i 4}$ & $\mathrm{~S}_{i 5}$ & Total & Rank \\
\hline Candidate-1 & 0.077 & 0.109 & 0.105 & 0.143 & 0.435 & 1 \\
Candidate-4 & 0.050 & 0.093 & 0.127 & 0.133 & 0.404 & 3 \\
Candidate-8 & 0.084 & 0.068 & 0.124 & 0.167 & 0.442 & 4 \\
Candidate-10 & 0.053 & 0.074 & 0.098 & 0.159 & 0.384 & 5 \\
Candidate-11 & 0.063 & 0.105 & 0.096 & 0.134 & 0.398 & 2 \\
\hline
\end{tabular}

\subsection{Discussion}

In the above case study the capability of fifteen candidates has been studied. Amongst them five candidates are selected and ranked according to their performance. The research study also demonstrates step-by-step elimination or selection of their candidature. The suitable wind turbine service technicians are enlisted who satisfy all the criteria mentioned in Table 2 better. For example, Candidate-3 satisfactorily scored in Test 2, 3 and 4, but failed miserably in Test 5 and goes out of Rank. Again Candidate 3 did not score the $50 \%$ of the desired level, where his/her overall performance is satisfied. This is one of the benefits in ARAS method that it is able to compare with the desired value.

\section{Now MOORA method is applied for the process of selection}

\subsection{Test 1}

In the same case study for an esteemed wind farm 15 numbers of candidates are applied for the post of Wind Turbine Technician. They are appeared for Test 1 . The decision weights of the criteria for selection of the best candidate are calculated using formula 6 and 7 and the result is tabulated in Table 9. The decision matrix for the Test 1 , in Linguistic term is tabulated in Table 9. The de-fuzzyfied BNP numbers and normalized decision matrix and weighted normalized decision matrix is calculated by using equation 2, 5 and 8 . Next the sum of benefit criteria measures $\left(S_{B}\right)$ and the sum of non-benefit 
criteria measures $\left(S_{N B}\right)$ are calculated by using equation 9 and 10 respectively. As all the criteria are beneficiaries in nature so the values of $S_{N B}$ are zero for all the alternatives.

Table 9

Decision Weights of the Criteria for Test-1

\begin{tabular}{|c|c|c|c|c|c|}
\hline Criteria & Subcriteria & L.T & T.F.N & $w_{j}$ & $q_{j}$ \\
\hline & C11 Traveling ability & $\mathrm{H}$ & $0.7,0.9,1.0$ & 0.867 & 0.248 \\
\hline & C12 Climbing Ability & $\mathrm{VH}$ & $0.9,1.0,1.0$ & 0.967 & 0.276 \\
\hline \multirow[t]{2}{*}{ C1 Physical Ability } & C13 Ability to perform strenuous job & VH & $0.9,1.0,1.0$ & 0.967 & 0.276 \\
\hline & C14 Weight lifting \& moving ability & $\mathrm{MH}$ & $0.5,0.7,0.9$ & 0.700 & 0.200 \\
\hline
\end{tabular}

Next the values of composite score are calculated by using equation 11 and ranking of the alternatives are tabulated in Table 10. Now the preliminary selection/elimination is performed on the basis of the composite score. In this study the candidates whose composite score is below 0.200 gets eliminated.

Table 10

Decision Matrix in Linguistic Term and Result for Test -1

\begin{tabular}{lllllccccc}
\hline \multicolumn{1}{c}{ Linguistic Terms } & & & & \\
\hline Candidates & C11 & C12 & C13 & C14 & $S_{B}$ & $S_{N B}$ & CS $_{i}$ & Rank & Qualified \\
Candidate-1 & G & MG & G & VG & 0.300 & 0 & 0.300 & 5 & $\sqrt{ }$ \\
Candidate-2 & M & MP & M & VP & 0.126 & 0 & 0.126 & 14 & \\
Candidate-3 & VG & G & VG & G & 0.329 & 0 & 0.329 & 3 & $\sqrt{ }$ \\
Candidate-4 & M & G & MP & M & 0.195 & 0 & 0.195 & 11 & \\
Candidate-5 & MP & M & G & G & 0.224 & 0 & 0.224 & 9 & $\sqrt{ }$ \\
Candidate-6 & VG & M & G & VG & 0.289 & 0 & 0.289 & 6 & $\sqrt{ }$ \\
Candidate-7 & P & MG & M & M & 0.166 & 0 & 0.166 & 12 & \\
Candidate-8 & G & VG & G & VG & 0.327 & 0 & 0.327 & 4 & $\sqrt{ }$ \\
Candidate-9 & MG & G & MG & M & 0.253 & 0 & 0.253 & 7 & $\sqrt{ }$ \\
Candidate-10 & VG & MG & M & VP & 0.207 & 0 & 0.207 & 10 & $\sqrt{ }$ \\
Candidate-11 & G & MG & M & MG & 0.245 & 0 & 0.245 & 8 & $\sqrt{ }$ \\
Candidate-12 & P & VP & MP & G & 0.106 & 0 & 0.106 & 15 & \\
Candidate-13 & VG & VG & G & VG & 0.336 & 0 & 0.336 & 1 & $\sqrt{ }$ \\
Candidate-14 & G & VG & VG & G & 0.330 & 0 & 0.330 & 2 & $\sqrt{ }$ \\
Candidate-15 & MP & MP & M & M & 0.141 & 0 & 0.141 & 13 & \\
\hline
\end{tabular}

\subsection{Test 2}

The qualified candidates of Test- 1 are appeared for Test 2 . The decision weight of the criteria for Test2 is tabulated in Table 11 below.

Table 11

Decision Weights of the Criteria for Test-2

\begin{tabular}{clcccc}
\hline \multicolumn{1}{c}{ Criteria } & \multicolumn{1}{c}{ Sub-criteria } & L.T & T.F.N & $w_{j}$ & $q_{j}$ \\
\hline \multirow{5}{*}{ C2 Basic Criteria } & C21Language Skill & MH & $0.5,0.7,0.9$ & 0.70 & 0.216 \\
& C22Mathematical Skill & $\mathrm{H}$ & $0.7,0.9,1.0$ & 0.87 & 0.268 \\
& C23Reasoning Ability & $\mathrm{MH}$ & $0.5,0.7,0.9$ & 0.70 & 0.216 \\
& C24Computer Knowledge & $\mathrm{VH}$ & $0.9,1.0,1.0$ & 0.97 & 0.299 \\
\hline \multicolumn{2}{c}{} & & & $\Sigma w_{j}=3.23$ & $\Sigma \mathrm{q}_{j}=1.000$ \\
\hline
\end{tabular}


The decision matrix for the Test 2, in Linguistic term and result are tabulated in Table 12. In Test 2, there is no elimination.

Table 12

Decision Matrix in Linguistic Term and Result for Test -2

\begin{tabular}{lccccccc}
\hline \multicolumn{7}{c}{ Linguistic Terms } & \\
Candidates & C21 & C22 & C23 & C24 & $S_{B}$ & $S_{N B}$ & CS $_{i 2}$ \\
Candidate-1 & G & VG & G & VG & 0.412 & 0 & 0.412 \\
Candidate-3 & VG & G & VG & MG & 0.385 & 0 & 0.385 \\
Candidate-5 & M & P & M & MG & 0.207 & 0 & 0.207 \\
Candidate-6 & MP & M & P & M & 0.168 & 0 & 0.168 \\
Candidate-8 & VP & M & M & M & 0.177 & 0 & 0.177 \\
Candidate-9 & G & VG & G & G & 0.399 & 0 & 0.399 \\
Candidate-10 & VG & G & VG & G & 0.407 & 0 & 0.407 \\
Candidate-11 & G & VG & G & VG & 0.412 & 0 & 0.412 \\
Candidate-13 & M & MP & P & M & 0.164 & 0 & 0.164 \\
Candidate-14 & P & M & M & P & 0.139 & 0 & 0.139 \\
\hline
\end{tabular}

\subsection{Test 3}

After Test 2, the candidates are appeared for Test 3. The decision weight of the criteria for selection for Test-3 is tabulated in Table 13.

Table 13

Decision Weights of the Criteria For Test-3

\begin{tabular}{cccccc}
\hline \multicolumn{1}{c}{ Criteria } & Subcriteria & L.T & T.F.N & $w_{j}$ & $q_{j}$ \\
\hline \multirow{4}{*}{ C3 Technical Criteria } & C31 Technical Knowledge \& Problem Sensitivity & VH & $0.9,1.0,1.0$ & 0.97 & 0.382 \\
& C32 Material management & MH & $0.5,0.7,0.9$ & 0.70 & 0.276 \\
& C33 Safety & H & $0.7,0.9,1.0$ & 0.87 & 0.342 \\
\hline
\end{tabular}

The decision matrix for the Test 3, in Linguistic term and the result are tabulated in Table 14. Now the preliminary selection/elimination is performed on the basis of the aggregating composite score of Test 2 and Test 3 . In this study the candidates whose composite score below 0.5 is eliminated.

Table 14

Decision Matrix in Linguistic Term and TFN Term for Test -3

\begin{tabular}{|c|c|c|c|c|c|c|c|c|c|}
\hline \multicolumn{4}{|c|}{$\begin{array}{c}\text { Linguistic } \\
\text { Terms } \\
\end{array}$} & \multirow[b]{2}{*}{$S_{B}$} & \multirow[b]{2}{*}{$S_{N B}$} & \multirow[b]{2}{*}{$\mathrm{CS}_{i 3}$} & \multirow[b]{2}{*}{$\mathrm{CS}_{i(2+3)}$} & \multirow[b]{2}{*}{ Rank } & \multirow[b]{2}{*}{ Qualified } \\
\hline Candidates & $\mathrm{C} 31$ & C32 & C33 & & & & & & \\
\hline Candidate-1 & VG & VG & $\mathrm{G}$ & 0.403 & 0 & 0.403 & 0.816 & 1 & $\sqrt{ }$ \\
\hline Candidate-3 & VG & $\mathrm{MG}$ & M & 0.315 & 0 & 0.315 & 0.700 & 3 & $\sqrt{ }$ \\
\hline Candidate-5 & $\mathrm{G}$ & $\mathrm{M}$ & M & 0.276 & 0 & 0.276 & 0.483 & 9 & \\
\hline Candidate- 6 & MG & G & G & 0.349 & 0 & 0.349 & 0.516 & 6 & $\sqrt{ }$ \\
\hline Candidate- 8 & MG & VG & G & 0.360 & 0 & 0.360 & 0.537 & 5 & $\sqrt{ }$ \\
\hline Candidate-9 & $\mathrm{M}$ & MG & M & 0.240 & 0 & 0.240 & 0.639 & 4 & $\sqrt{ }$ \\
\hline Candidate-10 & $\mathrm{P}$ & MP & MP & 0.103 & 0 & 0.103 & 0.510 & 7 & $\sqrt{ }$ \\
\hline Candidate-11 & VG & $\mathrm{G}$ & MG & 0.366 & 0 & 0.366 & 0.778 & 2 & $\sqrt{ }$ \\
\hline Candidate-13 & $\mathrm{P}$ & M & $\mathrm{G}$ & 0.214 & 0 & 0.214 & 0.379 & 10 & \\
\hline Candidate-14 & $\mathrm{G}$ & $\mathrm{G}$ & $\mathrm{MG}$ & 0.350 & 0 & 0.350 & 0.488 & 8 & \\
\hline
\end{tabular}




\subsection{Test 4}

Next the qualified candidates are to be appeared for a general interview (Test 4). Three selectors are appointed to conduct the selection process. The decision weight of the criteria for selection for Test 4 is tabulated in Table 15.

Table 15

Decision Weights of the Criteria for Test-4

\begin{tabular}{|c|c|c|c|c|c|}
\hline Criteria & Subcriteria & L.T & T.F.N & $w_{j}$ & $q_{j}$ \\
\hline \multirow{7}{*}{$\begin{array}{l}\text { Personal } \\
\text { Quality C4 }\end{array}$} & C41 Educational Qualification & $\mathrm{H}$ & $0.70,0.90,1.00$ & 0.87 & 0.178 \\
\hline & C42 Past Experience & $\mathrm{VH}$ & $0.90,1.00,1.00$ & 0.97 & 0.199 \\
\hline & C43 Leadership Quality & MH & $0.50,0.70,0.90$ & 0.70 & 0.144 \\
\hline & C44 Ability to work in a team & $\mathrm{H}$ & $0.70,0.90,1.00$ & 0.87 & 0.178 \\
\hline & C45 Self confidence & VH & $0.90,1.00,1.00$ & 0.97 & 0.199 \\
\hline & C46 Integrity and Honesty & M & $0.30,0.50,0.70$ & 0.50 & 0.103 \\
\hline & & & & $\sum w_{j}=4.8$ & $\Sigma \mathrm{q}_{j}=1.000$ \\
\hline
\end{tabular}

The group decision matrix for the Test 4, in Linguistic term is tabulated in Table 16. Now the preliminary selection/elimination is performed on the basis of the composite score. Here the candidates whose composite score is below 0.3 are eliminated and is shown in Table 17.

Table 16

Group Decision Matrix for Test -4 in Linguistic Term

\begin{tabular}{lccccccc}
\hline Candidates & Decision Makers & C 41 & C 42 & C 43 & C 44 & C 45 & C 46 \\
\hline \multirow{3}{*}{ Candidate-1 } & DM 1 & G & M & MG & G & MG & M \\
& DM 2 & G & G & MG & MG & M & M \\
& DM 3 & G & G & M & M & M & MG \\
\hline \multirow{3}{*}{ Candidate-3 } & DM 1 & G & M & G & G & MG & M \\
& DM 2 & VG & MG & VG & G & G & MG \\
& DM 3 & G & MG & MG & MG & MG & M \\
\hline \multirow{3}{*}{ Candidate-6 } & DM 1 & G & G & G & G & G & G \\
& DM 2 & VG & MG & MG & G & VG & MG \\
& DM 3 & G & G & M & MG & G & MG \\
\hline \multirow{3}{*}{ Candidate-8 } & DM 1 & G & M & G & MG & M & M \\
& DM 2 & M & M & MG & M & MG & M \\
& DM 3 & M & MG & MG & M & MP & MG \\
\hline \multirow{3}{*}{ Candidate-9 } & DM 1 & VG & G & VG & G & M & MG \\
& DM 2 & G & G & G & G & MG & M \\
\hline \multirow{3}{*}{ Candidate-10 } & DM 3 & G & G & G & VG & MG & M \\
& DM 1 & M & MG & MG & M & M & MG \\
& DM 2 & G & G & M & M & M & MG \\
Candidate-11 & DM 3 & M & MG & M & P & MP & MP \\
& DM 1 & G & G & MG & M & MG & M \\
& DM 2 & VG & G & MG & MG & M & M \\
\hline & DM 3 & G & MG & M & MP & P & MG \\
\hline
\end{tabular}

Table 17

Test-4 Result

\begin{tabular}{lccccc}
\hline Candidates & $S_{B}$ & $S_{N B}$ & $\mathrm{CS}_{i 4}$ & Rank & Qualified \\
\hline Candidate-1 & 0.348 & 0 & 0.348 & 4 & $\sqrt{ }$ \\
Candidate-3 & 0.387 & 0 & 0.387 & 3 & $\sqrt{ }$ \\
Candidate-6 & 0.421 & 0 & 0.421 & 1 & $\sqrt{ }$ \\
Candidate-8 & 0.301 & 0 & 0.301 & 6 & $\sqrt{ }$ \\
Candidate-9 & 0.408 & 0 & 0.408 & 2 & $\sqrt{ }$ \\
Candidate-10 & 0.281 & 0 & 0.281 & 7 & \\
Candidate-11 & 0.325 & 0 & 0.325 & 5 & $\sqrt{ }$ \\
\hline
\end{tabular}




\subsection{Test 5}

Lastly the qualified candidates are appeared for medical fitness test (Test 5). The decision weights of the criteria for selection of the best candidate are calculated using formula 3.24 and 3.25 and the result is tabulated in Table 18. The decision matrix for the Test 5, in Linguistic term and result of the test are tabulated in Table 19. Now the preliminary selection/elimination is performed on the basis of the composite score. In this study the candidates whose composite score below 0.300 gets eliminated.

Table 18

Decision Weights of the Criteria for Test-5

\begin{tabular}{clcccc}
\hline \multicolumn{1}{c}{ Criteria } & \multicolumn{1}{c}{ Subcriteria } & L.T & T.F.N & $w_{j}$ & $q_{j}$ \\
\hline \multirow{5}{*}{ C5 Medical } & C51 General health & $\mathrm{H}$ & $0.7,0.9,1.0$ & 0.87 & 0.160 \\
& C52 Diabetic fitness & $\mathrm{VH}$ & $0.9,1.0,1.0$ & 0.97 & 0.178 \\
Criteria & C53 Neuro-fitness & $\mathrm{H}$ & $0.7,0.9,1.0$ & 0.87 & 0.160 \\
& C54 Fitness of cardiovascular system & $\mathrm{H}$ & $0.7,0.9,1.0$ & 0.87 & 0.160 \\
& C55 Respiratory fitness & $\mathrm{H}$ & $0.7,0.9,1.0$ & 0.87 & 0.160 \\
& C56 Visual Acuity & $\mathrm{M}$ & $0.3,0.5,0.7$ & 0.50 & 0.092 \\
& C57 Hearing & $\mathrm{M}$ & $0.3,0.5,0.7$ & 0.50 & 0.092 \\
\hline
\end{tabular}

\section{Table 19}

Decision Matrix for Test -5 in Linguistic Term

\begin{tabular}{lccccccccccccc}
\hline Candidates & C51 & C52 & C53 & C54 & C55 & C56 & C57 & $S_{B}$ & $S_{N B}$ & CS $_{i 5}$ & Rank & Qualified \\
\hline Candidate-1 & M & G & VG & G & G & M & G & 0.418 & 0 & 0.418 & 3 & $\sqrt{ }$ \\
Candidate-3 & MG & P & MP & MP & M & G & G & 0.249 & 0 & 0.249 & 6 & \\
Candidate-6 & G & G & M & G & G & M & MG & 0.394 & 0 & 0.394 & 4 & $\sqrt{ }$ \\
Candidate-8 & G & G & MG & M & MG & M & M & 0.359 & 0 & 0.359 & 5 & $\sqrt{ }$ \\
Candidate-9 & VG & VG & G & VG & G & G & G & 0.481 & 0 & 0.481 & 1 & $\sqrt{ }$ \\
Candidate-11 & G & G & G & G & G & G & G & 0.457 & 0 & 0.457 & 2 & $\sqrt{ }$ \\
\hline
\end{tabular}

\section{Discussion}

Then the final merit list of five suitable candidates is prepared on the basis of composite score in all the tests. The candidate, who will compose all-round performance stand first in merit list, is tabulated in Table 20. In the above case study the capability of fifteen candidates has been studied initially by applying ARAS - F and subsequently by MOORA - F. Amongst them five candidates are selected and ranked according to their performance. This study also demonstrates step-by-step elimination or selection of their candidature. A suitable wind turbine technician is chosen who satisfies all the criteria mentioned in Table 3. It has been observed that applying both the techniques the list of candidates in the merit list are same and the candidate 1 and candidate 11 ranks $1^{\text {st }}$ and $2^{\text {nd }}$ in both the both the techniques.

Table 20

Final Result

\begin{tabular}{lcccccc}
\hline Candidates & $\mathrm{CS}_{i 1}$ & $\mathrm{CS}_{i(2+3)}$ & $\mathrm{CS}_{i 4}$ & $\mathrm{CS}_{i 5}$ & Total Composite score & Rank \\
\hline Candidate-1 & 0.300 & 0.816 & 0.348 & 0.418 & 1.882 & 1 \\
Candidate-6 & 0.289 & 0.516 & 0.421 & 0.394 & 1.620 & 4 \\
Candidate- 8 & 0.253 & 0.537 & 0.301 & 0.359 & 1.450 & 5 \\
Candidate-9 & 0.253 & 0.639 & 0.408 & 0.481 & 1.782 & 3 \\
Candidate-11 & 0.245 & 0.778 & 0.325 & 0.457 & 1.805 & 2 \\
\hline
\end{tabular}




\section{Conclusion}

The Fuzzy MCDM methodology can effectively signify the ambiguity and vagueness that are natural in any modern industry. The technique is quite flexible since criteria weights and alternatives considered can be replaced by any method for ranking fuzzy numbers. Fuzzy ARAS under group decision making is employed for recruiting technicians to form a merit list since it is beneficial in converting qualitative information into quantitative parameters. It is then validated using Fuzzy MOORA which corroborates the previous merit list. Contemporary approaches identify that selection of human resources is a multifaceted process that entail a considerable amount of ambiguity and prejudice which is a serious concern for candidate's qualms of career life. One of the principal objectives of Human Resource Development department is to employ the organization with the right workforce at the right time and in the right place. Human resource is a vital component and the overall performance of companies depends upon the way it is put to use. To solve the complexity and uncertainty evolved due to different conflicting criteria and different views of different decision makers of the proposed model, ARAS method is used, based on linguistic parameters and the fuzzy set theory. The method can assist human resources managers arrive at better decisions by selecting technicians through a process that takes into account organizational goals as well as technicians' qualities. Additionally, selection of employees can be seen as part of an integrated career management system in the organization.

The solutions will set a benchmark for the personnel management department of power generating industries to recruit suitable employees for better service to utilize their resources for better customer satisfaction. There is a scope for future researchers to analyze the model in different industries under different environment.

\section{Acknowledgement}

The authors would like to thank the anonymous referees for constructive comments on earlier version of this paper.

\section{References}

Afshari, A., Mojahed, M., \& Yusuff, R.M. (2010). Simple Additive Weighting approach to Personnel Selection problem, International Journal of Innovation, Management and Technology, 1(5), 511515.

Agarwal, R. (2013). Selection of IT personnel through hybrid multi-attributes AHP-FLP approach. International Journal of Soft Computing and Engineering, 2(6), 11-17.

Baležentis, A., Baležentis, T., \& Brauers, W. K. M. (2012). MultiMOORA-FG: A multi-objective decision making method for linguistic reasoning with an application to personnel selection. Informatica, 23(2), 173-190.

Blanco, M.I., \& Rodrigues, G. (2009), Direct employment in the wind energy sector: An EU study,.Energy Policy, 37(8), 2847-2857.

Bobrow, W. (2003). Personnel Selection and Assessment. The California Psychologist, 14-15.

Chatterjee, N. C., \& Bose, G. K. (2013). Selection of vendors for wind farm under fuzzy MCDM environment. International Journal of Industrial Engineering Computations, 4(4), 535 - 546.

Chen, C.T. (2000). Extensions of the TOPSIS for group decision-making under fuzzy environment. Fuzzy Sets and Systems, 114, 1-9.

Chen, M. F., Tzeng, G. H., \& Tang, T. I. (2005). Fuzzy MCDM approach for evaluation of expatriate assignments. International Journal of Information Technology \& Decision Making, 4(02), 277-296.

Chen, C. T., Pai, P. F., \& Hung, W. Z. (2011). Applying and Knowledge Map in Personnel Selection. Asia Pacific Management Review, 16(4), 491-502. 
Dadelo, S., Turskis, Z., Zavadskas, E., \& Dadeliene, R. (2012). Multiple Criteria Assessment of Elite Security Personal on the Basis of ARAS and Expert Methods. Economic Computation \& Economic Cybernetics Studies \& Research, 46(4), 65-88.

Datta, S., Beriha, G. S., Patnaik, B., \& Mahapatra, S. S. (2009). Use of compromise ranking method for supervisor selection: A multi-criteria decision making (MCDM) approach. International Journal of Vocational and Technical Education, 1(1), 7-13.

Ding, J. F. (2012). Using fuzzy MCDM model to select middle managers for global shipping carrierbased logistics service pProviders. WSEAS Transactions On Systems, 3(11), 85-94.

Dursun, M., \& Karsak, E. E. (2010). A fuzzy MCDM approach for personnel selection. Expert Systems with Applications, 37, 4324-4330.

El-Santawy, M. F. (2012). A VIKOR method for solving personnel training selection problem. International Journal of Computing Science, 1(2), 9-12.

Gibney, R., \& Shang, J. (2007). Decision making in academia: A case of the dean selection process. Mathematical and Computer Modeling, 46(7-8), 1030-1040.

Güngör, Z., Serhadlıŏlu, G., \& Kesen, S. E. (2009). A fuzzy AHP approach to personnel selection problem, Applied Soft Computing, 9, 641-646.

Haghighi, M., Zowghi, M., \& Ansari, M. (2012). A fuzzy multiple attribute decision making (MADM) approach for employee evaluation and selection process. American Journal of Scientific Research, $58,75-84$.

Hamilton, J., \& Liming, D. (2010). Careers in wind energy. U.S. Bureau of Labor Statistics, 1-18.

Karsak, E. E. (2001). Personnel selection using a fuzzy MCDM approach based on ideal and anti-ideal solutions. Lecture Notes in Economics and Mathematical Systems, 507, 393-402.

Kelemenis, A.M., \& Askounis, D. Th. (2009). An extension of fuzzy TOPSIS for personnel selection, In Proceedings of the 2009 IEEE International Conference on Systems, Man, and Cybernetics San Antonio, 4704-4709.

Kelemenis, A, Ergazakis, K., \& Askounis, D. (2011), Support managers' selection using an extension of fuzzy TOPSIS, Expert Systems with Applications, 38(3), 2774-2782.

Keršulienè. V., \& Turskis, Z. (2011), Integrated fuzzy multiple criteria decision making model for architect selection, Technological and Economic Development of Economy, 17(4), 645-666.

Liang, J., \& Pang, J. (2012). Evaluation of the results of multi-attribute group decision-making with linguistic information, Omega, 40, 294-301.

Liang, G. S., \& Wang, M. J. J. (1994). Personnel selection using fuzzy MCDM algorithm. European Journal of Operational Research, 78(1), 22-33.

Pramanik, S., \& Mukhopadhyaya, D. (2011). Grey Relational Analysis based Intuitionistic Fuzzy Multi-criteria Group Decision-making Approach for Teacher Selection in Higher Education. International Journal of Computer Applications, 34(10), 21-29.

Ramadan, M. Z. (2009). Effective staff selection tool: Fuzzy numbers and memetic algorithm based approach. International Journal of Engineering \& Technology, 9(10), 54-65.

Robertson, I. T., \& Smith, B. (2001). Personnel selection. Journal of Occupational and Organizational Psychology, 74, 441-472.

Rouyendegh, B.D., \& Erkan, T.E., (2013). An Application of the Fuzzy ELECTRE method for academic staff selection. Human Factors and Ergonomics in Manufacturing \& Service Industries, 23(2), 107-115.

Turskis, Z., \& Zavadskas, E. K. (2010). A new fuzzy additive ratio assessment method (ARAS-F). Case study: The analysis of fuzzy multiple criteria in order to select the logistic centers location. Transport, 25(4), 423-432.

Zadeh, L.A. (1965). Fuzzy sets. Information and Control, 8, 338-353.

Zavadskas, E.K., Turskis, Z., Tamosaitiene, J., \& Marina, V. (2008). Selection of Construction Project Managers by Applying COPRAS-G Method, In The 8th International Conference of Reliability and Statistics in Transportation and Communication - 2008, 344-350. 
Zavadskas, E. K., \& Turskis, Z. (2010). A new additive ratio assessment (ARAS) method in multicriteria decision-making. Technological and Economic Development of Economy, 16(2), 159172.

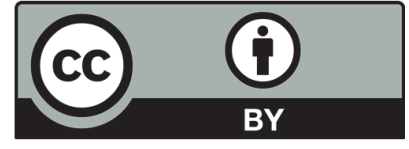

(C) 2016 by the authors; licensee Growing Science, Canada. This is an open access article distributed under the terms and conditions of the Creative Commons Attribution (CC-BY) license (http://creativecommons.org/licenses/by/4.0/). 